Vittalle - Revista de Ciências da Saúde v. 30, n. 1 (2018) 26-35

\title{
Vittalle
}

ISSN 2177-7853

\section{A aceitação da terapia homeopática em hospitais da Universidade Federal do Rio de Janeiro}

\author{
Giovanna Menezes Iozzi ${ }^{\mathrm{a},{ }^{*}} ;$ Rosanna Iozzi da Silva ${ }^{\mathrm{c}}$; Fortune Homsani ${ }^{\mathrm{a}}$; Adriana Passos \\ Oliveira ${ }^{\mathrm{a}}$; Marcia Alves Marques Capella ${ }^{\mathrm{b}}$; Carla Holandino, ${ }^{\mathrm{a}, *}$
}

aa Laboratório Multidisciplinar de Ciências Farmacêuticas e Laboratório de Pesquisa \& Desenvolvimento de Práticas Integrativas e Complementares, Departamento de Fármacos e Medicamentos, Faculdade de Farmácia,

UFRJ, Rio de Janeiro, RJ, Brasil

${ }^{\mathrm{b}}$ Instituto de Biofísica Carlos Chagas Filho, UFRJ, Rio de Janeiro, RJ, Brasil

${ }^{\mathrm{c}}$ Secretaria Municipal de Saúde do Rio de Janeiro, Rio de Janeiro, RJ, Brasil

\section{Histórico do Artigo \\ Recebido em: \\ 03/11/2017 \\ Aceito em: \\ 30/03/2018 \\ Palavras-chave: \\ Homeopatia; \\ hospitais universitários; \\ PNPIC; HUCFF; IPPMG}

Key-words:

University hospitals;

PNPIC; HUCFF;

IPPMG; homeopathy.

\section{RESUMO}

Nos últimos dez anos, tem-se verificado o aumento do interesse pela terapia homeopática devido a sua inclusão no SUS, oficializada na "Política Nacional de Práticas Integrativas e Complementares" (PNPIC). A Universidade Federal do Rio de Janeiro (UFRJ) possui dois hospitais universitários de grande importância no Sistema Único de Saúde (SUS): o Hospital Universitário Clementino Fraga Filho (HUCFF) e o Instituto de Puericultura e Pediatria Martagão Gesteira (IPPMG). O presente estudo avaliou, ao todo, a opinião de 142 médicos, sendo 110 do HUCFF e 32 do IPPMG, sobre a homeopatia, através de um questionário específico. Após coleta e tratamento estatístico dos dados, os resultados indicaram que apenas 14 médicos já tinham indicado e/ou utilizado a terapia homeopática anteriormente, apesar da inexistência de ambulatório de homeopatia em ambos os hospitais avaliados. A análise dos questionários evidenciou, também, que a maioria dos médicos entrevistados, além de desconhecerem a terapia homeopática, acham irrelevante que o serviço de homeopatia seja oferecido nesses hospitais. Dessa forma, verificamos a necessidade de inclusão da homeopatia na graduação de medicina da UFRJ com a ampliação do currículo dos médicos da UFRJ, como já é feito na UNIRIO e na UFF, e de mapear a percepção dos profissionais da saúde acerca da homeopatia, fornecendo dados que favoreceram a implantação da homeopatia na Atenção Básica à Saúde, como previsto na PNPIC.

The acceptance of homeopathic therapy in hospitals of the Federal University of Rio de Janeiro

\section{ABSTRACT}

The Federal University of Rio de Janeiro (UFRJ) has two teaching hospitals of great importance for the Brazilian Public Health Program (SUS): Hospital Clementino Fraga Filho (HUCFF) and Instituto de Puericultura e Pediatria Martagão Gesteira (IPPMG). In the last ten years, we could detect an increasing interest for homeopathic therapy, due to its inclusion in SUS, which is managed by the "National Policy for Integrative and Complementary Practices (PNPIC)". The present study evaluated physicians' opinions about Homeopathy through a questionnaire with 142 professionals, being 110 from HUCFF and 32 from IPPMG in both hospitals. After the collection and statistical treatment of data, the results showed only 14 physicians had previously prescribed homeopathic medicines, even without homeopathic ambulatory. A lack of knowledge about homeopathy was detected and the majority of physicians do not see any reason to offer this therapeutic service in these hospitals. Eventually, it is possible to claim how important it is to include Homeopathy teaching in the curriculum to increase the knowledge of Medicine students about it, and to evaluate the perception of health professionals about homeopathy to favor its inclusion in the Brazilian Public Health Program.

\footnotetext{
*Autores correspondentes: giovanna.iozzi@gmail.com (G. M. Iozzi) ou cholandino@ gmail.com (C. Holandino).
} 


\section{Introdução}

A homeopatia é um sistema médico complexo, de caráter holístico, baseada no princípio vitalista e no uso da lei dos semelhantes (1). Medicamento homeopático, segundo a farmacopeia homeopática brasileira (FHB), é toda apresentação farmacêutica destinada a ser ministrada segundo o princípio da similitude, com finalidade preventiva e terapêutica, obtida pelo método de diluições seguidas de sucussões (agitação) e/ou triturações sucessivas. Para sua confecção, empregam-se substâncias dos reinos animal, vegetal e mineral sob as recomendações da farmacotécnica homeopática (2). Mesmo diante do crescimento e do reconhecimento da terapia homeopática no Brasil, muitos ambientes públicos de saúde ainda não oferecem os serviços homeopáticos, assim como muitas faculdades de medicina, responsáveis por formar um dos profissionais capacitados a prescrever os medicamentos homeopáticos, não têm adotado o ensino de homeopatia em suas grades curriculares, fatos que vêm dificultando o crescimento da homeopatia no Sistema Único de Saúde (SUS) e no meio universitário. Dentre as 52 faculdades de medicina listadas pela Associação Brasileira de Educação Médica (Abem), apenas 17 oferecem atividades relacionadas à homeopatia. Das três universidades federais existentes na cidade do Rio de Janeiro (UFRJ, UNIRIO e UFF), somente a Universidade Federal do Rio de Janeiro (UFRJ) não oferece a disciplina em seu currículo (3). Em contrapartida, a Faculdade de Farmácia da UFRJ vem ocupando papel de destaque no cenário nacional no que diz respeito ao ensino da homeopatia tanto no âmbito da graduação quanto da pós-graduação.

A faculdade de medicina da UFRJ, além de contar com seus ambientes dentro do Centro de Ciências da Saúde (CSS), possui dois importantes hospitais universitários situados dentro da cidade universitária que são essenciais para a formação dos alunos. São eles: o Instituto de Puericultura e Pediatria Martagão Gesteira (IPPMG) e o Hospital Clementino Fraga Filho (HUCFF). O IPPMG é uma das construções mais antigas da cidade universitária e foi fundado em 1953. Desde a sua fundação, vem atendendo às finalidades de ensino e pesquisa na área pediátrica, a partir da assistência prestada à população (4). Assim como o IPPMG, o HUCFF funciona como referência no tratamento de diversas patologias de alta complexidade, sendo um centro de excelência em ensino, pesquisa e extensão da UFRJ (5).

Em 1988, no mesmo ano em que o SUS foi criado e a saúde passou a ser vista como direito social de toda a população, as práticas de medicina alternativas foram incorporadas como recurso terapêutico válido e eleitas como direito social (6). Em 2006, a partir do crescimento da prática homeopática, a homeopatia foi oficialmente inserida no Sistema Único de Saúde, através da Portaria Ministerial 971/2006 (7), denominada "Política Nacional de Práticas Integrativas e Complementares" (PNPIC). Nessa portaria, foram incluídas além da homeopatia, a medicina tradicional chinesa, a medicina antroposófica, as plantas medicinais e fitoterapia e o termalismo social/crenoterapia. Posteriormente, no ano de 2017, 14 novas práticas foram incorporadas ao sistema público de saúde, através da Portaria 849/2017, ampliando para 19 o número de PICS que podem ser ofertados nos municípios brasileiros (8).

A PNPIC prevê em suas diretrizes e metas o desenvolvimento de estratégias para a ampliação do conhecimento dos profissionais da saúde acerca dos fundamentos, potencial medicamentoso, custos e outras características da terapêutica homeopática, visando ao seu crescimento nas esferas do SUS (7). Sendo assim, o presente estudo teve como objetivo avaliar o conhecimento e a aceitação que os médicos, de dois hospitais de referência na cidade do Rio de Janeiro, possuem em relação à homeopatia. 


\section{Materiais e Métodos}

O trabalho realizado é um estudo transversal descritivo-quantitativo (9). O mesmo foi realizado em dois hospitais federais pertencentes à UFRJ, o Instituto de Puericultura e Pediatria Martagão Gesteira e o Hospital Clementino Fraga Filho, durante o período de junho de 2014 a outubro de 2014. Para composição da amostra, a mesma foi dividida em dois grupos: médicos do HUCFF e médicos do IPPMG. Foram selecionados para o estudo $15 \%$ do número total de médicos de cada hospital, totalizando 110 médicos do HUCFF e 32 médicos do IPPMG, tendo-se utilizado uma amostra por conveniência, onde foram entrevistados apenas os médicos que se dispuseram a participar do estudo.

Para a coleta das informações, utilizou-se um questionário, elaborado a partir das bases teóricas da homeopatia $(1,6)$ e o que é estabelecido pela PNPIC $(7,8)$, acrescido de parâmetros utilizados em outro questionário de pesquisa de caráter exploratório (9; (Quadro 1).

Quadro 1: Questionário utilizado nas entrevistas para coleta de dados dos médicos do HUCFF e do IPPMG.

\begin{tabular}{|c|c|c|c|c|}
\hline Perguntas & \multicolumn{4}{|c|}{ Possíveis Respostas } \\
\hline Gênero & \multicolumn{2}{|c|}{ masculino } & \multicolumn{2}{|l|}{ feminino } \\
\hline Idade & \multicolumn{4}{|l|}{ livre } \\
\hline Qual é o seu cargo? & staff & professor & residente & $\begin{array}{l}\text { aluno de } \\
\text { medicina }\end{array}$ \\
\hline Você sabe o que é homeopatia? & \multicolumn{2}{|l|}{ Sim } & \multicolumn{2}{|l|}{ Não } \\
\hline $\begin{array}{l}\text { Durante a faculdade, você teve } \\
\text { alguma aula ou algum conteúdo } \\
\text { sobre homeopatia? }\end{array}$ & \multicolumn{2}{|l|}{ Sim } & \multicolumn{2}{|l|}{ Não } \\
\hline Qual o princípio da homeopatia? & \multicolumn{4}{|l|}{ livre } \\
\hline Você já usou homeopatia? & \multicolumn{2}{|l|}{ Sim } & \multicolumn{2}{|l|}{ Não } \\
\hline Você usaria? & \multicolumn{2}{|l|}{ Sim } & \multicolumn{2}{|l|}{ Não } \\
\hline Por quê? & \multicolumn{4}{|l|}{ livre } \\
\hline Você atende no ambulatório? & \multicolumn{2}{|l|}{ Sim } & \multicolumn{2}{|l|}{ Não } \\
\hline Já atendeu? & \multicolumn{2}{|l|}{$\operatorname{sim}$} & \multicolumn{2}{|l|}{ Não } \\
\hline Qual a sua especialidade & \multicolumn{4}{|l|}{ livre } \\
\hline $\begin{array}{l}\text { Você já usou a homeopatia como } \\
\text { terapia complementar para seus } \\
\text { pacientes? }\end{array}$ & \multicolumn{2}{|l|}{$\operatorname{sim}$} & \multicolumn{2}{|l|}{ Não } \\
\hline Você usaria? & \multicolumn{2}{|l|}{$\operatorname{sim}$} & \multicolumn{2}{|l|}{ Não } \\
\hline Por que não? & \multicolumn{4}{|l|}{ livre } \\
\hline $\begin{array}{l}\text { Você seria a favor da existência } \\
\text { de um ambulatório homeopático } \\
\text { neste hospital? }\end{array}$ & $\operatorname{sim}$ & não & \multicolumn{2}{|l|}{ Indiferente } \\
\hline
\end{tabular}

Além do questionário, os entrevistados assinaram um Termo de Consentimento Livre e Esclarecido (TCLE), confeccionado conforme as determinações do Comitê de Ética em Pesquisas da UFRJ, sendo aprovado pelo mesmo comitê de ética em 25/09/2014 (CAEE 35626614.9.0000.5257).

O questionário continha 12 perguntas com intuito de mapear o conhecimento dos entrevistados com relação à homeopatia, à existência ou não de experiência clínica e à aceitabilidade quanto à criação de ambulatório de homeopatia em ambos os hospitais. (Quadro 1). Em média, as entrevistas duravam cerca de 2 minutos, quando, depois de 
orientado, o próprio médico respondia ao questionário. Foram convidados a participar voluntários que estivessem disponíveis nos ambulatórios do primeiro e do segundo andar do HUCFF, assim como aqueles presentes nas enfermarias de clínica médica, dermatologia, de doenças infecto-parasitárias, geriatria, psiquiatria, cardíaca e do centro cirúrgico. No IPPMG, foram convidados a participar os médicos do ambulatório, das enfermarias e da emergência do hospital. A escolha dos voluntários convidados para participar do estudo foi feita de forma aleatória, sem distinção de gênero ou idade, evitando qualquer preferência entre os voluntários selecionados.

Os dados coletados foram transferidos para um banco de dados e quantificados pelo Microsoft Office Excel 2007. A quantificação dos dados foi feita por tabulação (9). Os questionários foram enumerados e a informação obtida em cada resposta foi colocada em uma linha da tabela confeccionada.

\section{Resultados}

\subsection{Caracterização das Amostras}

No hospital HUCFF, a amostra continha 110 médicos e revelou que $57 \%$ da população entrevistada era de mulheres e $43 \%$ de homens. A média de idade dos médicos foi de 34 anos, porém a maioria dos entrevistados, 63\%, tinham entre 20 e 29 anos. A maioria, $68 \%$, era residente nos ambulatórios e nas enfermarias do HUCFF, seguido de $24 \%$ de médicos e $6 \%$ de professores.

A maioria dos médicos entrevistados do HUCFF, 78\%, atendia no ambulatório; o restante dos entrevistados, $22 \%$, disseram não realizar atendimento ambulatorial.

Dentre os médicos entrevistados do HUCFF, em relação aos seus locais de origem, 87\% eram do Estado do Rio de Janeiro; os outros 13\% eram oriundos de outros Estados, a saber: Alagoas, Bahia, Goiás, Minas Gerais, Pará, Paraíba, Paraná e Rio Grande do Sul. O Distrito Federal também foi representado. Dentre os entrevistados do HUCFF, $44,5 \%$ eram formados pela própria UFRJ, enquanto $55,5 \%$ vieram de 23 diferentes faculdades.

No hospital IPPMG, a amostra contendo 32 médicos revelou que $81 \%$ dos entrevistados era do gênero feminino, e apenas $19 \%$ era do gênero masculino. Apesar do maior número de médicos, 53\%, encontrar-se no intervalo entre 20 e 29 anos, a média de idade dos médicos do IPPMG foi de 36 anos.

A maioria dos entrevistados, 59\%, era residente do IPPMG, seguido de $38 \%$ de médicos e de 3\% de professores das diferentes unidades do IPPMG (ambulatório, enfermaria, emergência). A maioria dos médicos, $78 \%$ dos entrevistados, atendia no ambulatório e $22 \%$ dos entrevistados disseram não realizar atendimento ambulatorial.

Dentre os médicos entrevistados no IPPMG, apenas 1 não era do Estado do Rio de Janeiro. No IPPMG, $38 \%$ dos médicos receberam a formação acadêmica na própria UFRJ, enquanto $63 \%$ vieram de outras 8 faculdades.

\subsection{Dados Médicos}

Os resultados evidenciaram que 97\% dos médicos do IPPMG e 95\% dos médicos do HUCFF afirmaram conhecer a terapia homeopática (Figura 1). 


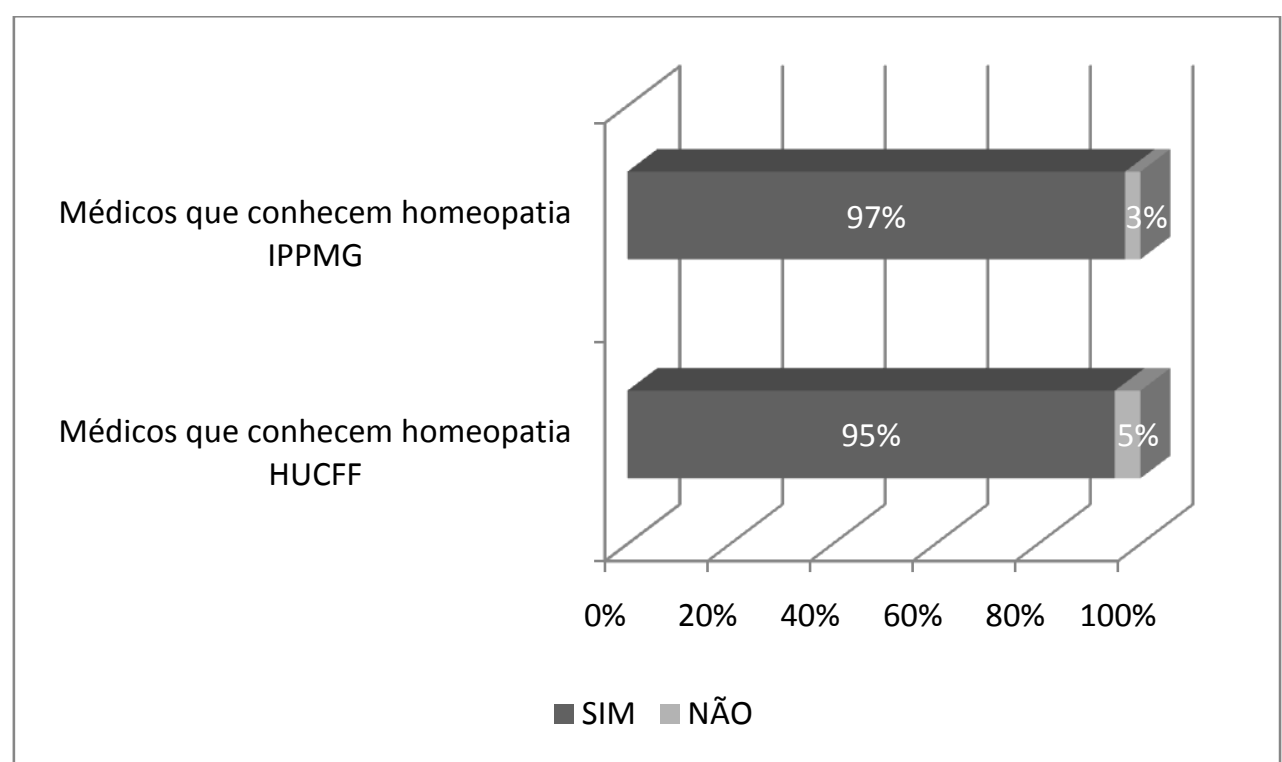

Figura 1: Porcentagem de médicos dos hospitais HUCFF e IPPMG que relataram conhecer ou não a terapia homeopática, num total de 110 entrevistados no HUCFF e 32 entrevistados no IPPMG.

Porém, foi possível detectar a falta de conhecimentos básicos e ainda em alguns casos, uma confusão acerca dos princípios que norteiam a terapêutica, quando os médicos foram questionados com a pergunta "Qual é o princípio da homeopatia?" (Tabela 1). As respostas obtidas foram classificadas em três grandes grupos: A) Não sei; B) Citou um dos cinco itens relacionados às bases da terapia homeopática (cura pela semelhança; medicamentos únicos; experimentação no homem são; doses infinitesimais; dinamização); C) Não citou nenhum dos cinco itens relacionados à terapia. A quantificação dos dados mostrou que 57\% dos entrevistados do HUCFF e $56 \%$ dos entrevistados do IPPMG não sabem nada sobre homeopatia ou responderam algo que não é relacionado com a terapia, enquanto apenas 35\% dos médicos do HUCFF e $43 \%$ dos médicos do IPPMG responderam ao questionamento com informações condizentes com a terapia homeopática.

Tabela 1: Número e porcentagem de médicos dos hospitais HUCFF e IPPMG em relação às respostas do questionamento "Qual é o princípio da homeopatia?".

\begin{tabular}{lllll} 
Hospitais & \multicolumn{2}{c}{ HUCFF } & \multicolumn{2}{c}{ IPPMG } \\
\hline Grupos & $\begin{array}{c}\text { Número de } \\
\text { entrevistados }\end{array}$ & Porcentagem & $\begin{array}{c}\text { Número de } \\
\text { entrevistados }\end{array}$ & Porcentagem \\
\hline Grupo A & 27 & $25 \%$ & 7 & $30 \%$ \\
\hline Grupo B & 38 & $35 \%$ & 10 & $43 \%$ \\
\hline Grupo C & 35 & $32 \%$ & 6 & $26 \%$ \\
\hline Branco & 10 & $9 \%$ & 9 & $39 \%$ \\
\hline Total & 110 & & 23 &
\end{tabular}

Em relação à faculdade que os médicos cursaram ter oferecido alguma disciplina, curso ou outro tipo de conteúdo sobre homeopatia, foram observados os seguintes resultados: dentre os alunos das 4 faculdades que possuem atividades relacionadas à homeopatia (UFF, UNIRIO, UFPB, UNB) (3), os alunos da UNB e UFPB disseram que não tiveram nenhum conteúdo sobre homeopatia; todos os alunos da UNIRIO disseram ter recebido algum conteúdo sobre a terapia homeopática durante a graduação e, dentre os alunos da UFF, houve uma divergência de respostas, quando alguns confirmaram a 
existência de conteúdo em suas grades curriculares, enquanto outros negaram. Finalmente, alguns alunos de faculdades que não possuem oficialmente atividades sobre homeopatia (UFAM, UFRJ, UniFOA, UNIGRANRIO) (3) disseram ter recebido algum conteúdo durante a graduação, sem maiores especificações.

Os médicos entrevistados também foram questionados se já haviam utilizado e/ ou indicado a terapia homeopática para seus pacientes e, de acordo com a resposta dada, foram questionados se poderiam vir a fazer essa indicação e/ou utilização (Tabela 2).

Tabela 2: Avaliação da utilização e/ou possível utilização da terapia pelos médicos dos hospitais HUCFF e IPPMG.

Você já usou a homeopatia como terapia complementar para seus pacientes?

\begin{tabular}{lccc}
\hline Hospitais & Sim & Não & Total \\
\hline HUCFF & $\begin{array}{c}3,6 \% \\
(4 \text { médicos })\end{array}$ & $\begin{array}{c}96,4 \% \\
(106 \text { médicos })\end{array}$ & 110 \\
\hline $\begin{array}{c}9,4 \% \\
\text { IPPMG }\end{array}$ & $\begin{array}{c}26,4 \% \\
(22 \text { médicos })\end{array}$ & 32 \\
\hline Você usaria? & & & \\
\hline Hospitais & Sim & Não & Total \\
\hline HUCFF & $26 \%$ & $74 \%$ & 106 \\
\hline IPPMG & $48 \%$ & $52 \%$ & 29
\end{tabular}

Dentro do HUCFF, apenas 3,6\% dos médicos já haviam utilizado a terapia homeopática com seus pacientes, enquanto no IPPMG 9,4\% dos médicos já havido feito essa utilização. Salientamos que, no IPPMG, o universo de entrevistados totalizou 32 voluntários, que foi um número aproximadamente 3 vezes menor do que o número de voluntários entrevistados no HUCFF.

Dentre os 106 médicos entrevistados do HUCFF que nunca tinham utilizado a terapia homeopática em seus pacientes, $26 \%$ relataram que poderiam vir a utilizar a terapêutica homeopática. Desses, $26 \%$ entrevistados, apenas 25\% dos médicos, pertenciam ao hospital de fato, pois, $71 \%$ eram residentes e $4 \%$ eram visitantes do hospital. Nossos resultados indicaram, ainda, que, neste universo de médicos do IPPMG entrevistados (36\% do quadro oficial e 64\% médicos residentes), $29 \%$ nunca tinham utilizado a terapia homeopática em seus pacientes; entretanto, $48 \%$ desses disseram que usariam ou indicariam a homeopatia para os seus pacientes.

Esses resultados suscitaram um questionamento feito aos entrevistados acerca do motivo da não utilização da homeopatia. Verificamos que as respostas fornecidas foram basicamente duas: os médicos do IPPMG responderam que a resistência ao uso se baseava na falta de conhecimento da terapêutica, a qual se refletiria na insegurança quanto à prescrição ("Por não conhecer ou por não se achar apto a prescrever"); em contrapartida, os médicos do HUCFF forneceram, como principal resposta, a falta de confiança na terapêutica ("Não acredito ou não acho que funcione").

Por último, os médicos foram questionados quanto à criação de um ambulatório homeopático nos respectivos hospitais. A pergunta feita foi: "Você seria a favor da existência de um ambulatório homeopático neste hospital?" (Figura 2). 


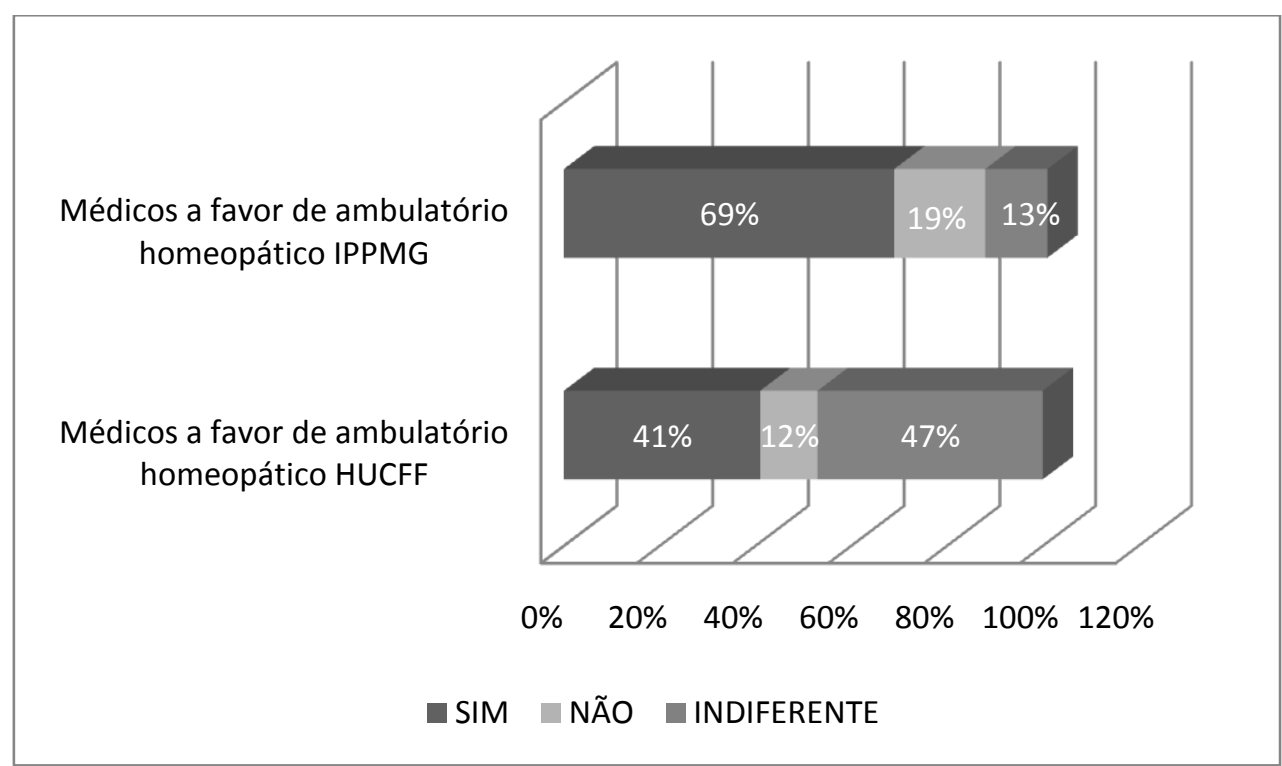

Figura 2: Porcentagem de médicos que se manifestaram a favor ou contra a criação de um ambulatório homeopático nos hospitais HUCFF e IPPMG, num total de 110 médicos entrevistados no HUCFF e 32 médicos entrevistados no IPPMG.

Pode-se observar que $69 \%$ dos entrevistados no IPPMG e $41 \%$ dos entrevistados no HUCFF se mostraram favoráveis à criação desse ambulatório. Dentre os $69 \%$ entrevistados dos IPPMG, 40\% eram médicos do hospital, 5\% eram professores e 55\% eram médicos residentes. Dentro os $41 \%$ entrevistados do HUCFF, $27 \%$ eram médicos do hospital, $9 \%$, professores, $62 \%$, médicos residentes e $2 \%$ não se identificaram.

\section{Discussão}

\subsection{Caracterização das Amostras}

Tanto no HUCFF quanto no IPPMG, pode-se observar uma grande presença feminina dentre os vários médicos entrevistados, confirmando a predominância do gênero feminino nas matrículas do Ensino Superior de medicina, desde 2001 (10). Outro dado também observado foi em relação à pediatria ser predominantemente exercida por mulheres no IPPMG, fato observado, anteriormente, por outros autores (10).

Nos dois hospitais, registramos uma pequena quantidade de professores entrevistados, devido à dificuldade de acesso a eles durante a realização da pesquisa. Adicionalmente, nos dois hospitais, a parcela de médicos que não atende nos ambulatórios, $22 \% \mathrm{em}$ ambos, em sua maioria foi representada por professores e médicos que já atenderam, em algum momento, no ambulatório dos respectivos hospitais, mas que, atualmente, apenas orientam alunos de residência. Vale ressaltar que apenas no HUCFF, parte dos $22 \%$ dos médicos que não realizam atendimento ambulatorial era residente de anestesiologia.

Pode-se observar que o HUCFF possui uma quantidade maior de médicos de outros Estados em relação ao IPPMG. Em ambos os hospitais, a maioria dos médicos não teve sua formação acadêmica na UFRJ, o que reforça o status de referência dos hospitais dentro no cenário da saúde e da formação de medicina do país.

\subsection{Dados Médicos}

Devido ao destaque que a homeopatia vem ocupando nas várias esferas da saúde pública brasileira desde a publicação da Política Nacional de Práticas Integrativas e 
Complementares (7), como esperado, observou-se que a maioria dos entrevistados disse conhecer a terapia homeopática (Figura 1).

As respostas obtidas pelos médicos quando perguntados sobre o princípio da homeopatia foram classificadas de acordo com definições da literatura $(1,2)$ que descrevem a homeopatia como uma prática médica e farmacêutica que se baseia na lei dos semelhantes (1). Da mesma forma, a definição de medicamento homeopático como um medicamento obtido pela técnica de dinamização (diluições seguidas de sucussões e/ou triturações), como definição farmacopeica (2). Mesmo com uma alta porcentagem de médicos afirmando conhecer a terapia homeopática (Figura 1), verificamos que a maioria deles desconhece as bases que a fundamentam (Tabela 1). Pode-se observar, também, que, quando comparados entre si, os médicos entrevistados do IPPMG demonstraram ter um conhecimento maior acerca da terapêutica, totalizando $43 \%$ dos entrevistados, em relação ao percentual de 35\% dos médicos do HUCFF (Tabela 1). Esse dado corrobora os resultados descritos na Figura 1, onde uma porcentagem menor de entrevistados relatou não conhecer a terapia no IPPMG em relação ao HUCFF.

Comparamos os resultados obtidos com os encontrados na literatura $(3,11)$, em que foi realizado um levantamento das faculdades que ofereciam atividades relacionadas à homeopatia e qual era essa atividade. Dentre as faculdades que possuem atividades relacionadas à homeopatia (UFF/ UNB/ UFPB/ UNIRIO (1)), a UNIRIO apresenta uma disciplina de caráter obrigatório na graduação, o que pode justificar o fato desses estudantes relatarem conhecimento mínimo acerca dos conteúdos relacionados à homeopatia. Os alunos da UFF apresentaram dois resultados ("sim" e "não"), isso pode ser facilmente compreendido, visto que a faculdades apresentam disciplina de caráter optativo, tornando a realização da disciplina uma escolha do aluno. Da mesma forma, UFF e UFPB também possuem uma disciplina de caráter optativo, o que explica a resposta negativa dos alunos dessas faculdades. A UNB diz fornecer conteúdo sobre a terapia dentro de outra disciplina, o que faz com que alunos menos atentos ao tema não percebam a presença do mesmo ou esqueçam que o mesmo foi contemplado em algum momento, sendo assim, compreensível o aluno entrevistado ter respondido ausência de conteúdo sobre homeopatia na graduação. Em relação às faculdades que não declararam ter conteúdo homeopático na graduação de forma oficial, a saber, UFAM, UFRJ, UniFOA, UNIGRANRIO, ressaltamos duas possíveis hipóteses para esse resultado encontrado: $1^{\mathrm{o}}$ : o tema ter sido abordado em apenas uma aula de outra disciplina; $2^{\circ}$ : o tema ter sido abordado como um subitem de outro tema, não sendo, dessa forma, apresentado de forma clara. Por isso, alguns desses alunos não registraram o conteúdo de maneira explícita.

Adicionalmente, estudo quantitativo recente acerca da oferta de disciplinas e cursos em PICs em seis instituições de Ensino Superior públicas no Estado do Rio de Janeiro (11) evidenciou que o ensino de PICS existe em 56 unidades de ensino, com maior concentração em cursos de Medicina, Farmácia e Enfermagem. Dentre as PICS, a Homeopatia, a Meditação e as Práticas Corporais têm sido lecionadas de maneira mais frequente, sinalizando o interesse de instituições de ensino no ensino das PICS, fato que pode vir a mudar, a curto prazo, o desconhecimento por parte dos profissionais de saúde registrado em nosso estudo.

Apesar de um pequeno número de médicos já ter utilizado e/ou indicado a terapia homeopática com seus pacientes (Tabela 2), observamos uma boa receptividade dos mesmos com relação à criação de um ambulatório homeopático. Vale ressaltar, porém, que a maioria dos médicos entrevistados nos dois hospitais que se mostraram a favor do ambulatório homeopático era residente no hospital e que o número de profissionais permanentes dos hospitais a favor de ambulatório homeopático foi pequeno. Além 
disso, dentre os vários especialistas entrevistados no HUCFF, os infectologistas foram os que mais se mostraram favoráveis à introdução da homeopatia como recurso terapêutico para o tratamento dos seus pacientes.

Considerando os dados obtidos em relação ao conhecimento homeopático (Figura 1); à quantidade de médicos que já utilizaram homeopatia como terapia complementar e integrativa (Tabela 2); aos médicos que usariam a terapia no tratamento dos seus pacientes e à possível criação de um ambulatório homeopático (Figura 2), pode-se sugerir que os médicos do IPPMG ou os pediatras, de uma forma geral, têm maior abertura para a terapia homeopática.

Além da percepção em relação aos pediatras, pode-se visualizar uma incoerência entre a oferta da terapia homeopática ser algo garantido através de uma política governamental específica $(7,8)$, e o serviço homeopático não ser oferecido nos hospitais e não ser bem aceito pelos profissionais entrevistados nos mesmos. Além disso, é preciso que se estimule a ampliação do ensino das PICS nas várias instituições brasileiras, incluindo a UFRJ, para que novos profissionais sejam alcançados, possibilitando o acesso ao conhecimento necessário para viabilizar a oferta da homeopatia, através da formação de recursos humanos dentro das universidades (11).

Em última instância, ressaltamos que o presente estudo apresenta algumas limitações como: acesso maior apenas aos médicos residentes, que, em geral, possuem pouca experiência profissional; a dificuldade de acesso aos professores e o curto período de tempo de realização desta pesquisa. Essas limitações apontam para a necessidade de novos levantamentos com tamanhos amostrais mais significativos que forneçam um mapeamento mais preciso.

\section{Considerações Finais}

O presente estudo nos permitiu caracterizar a população entrevistada dos médicos presentes em dois hospitais da UFRJ, o HUCFF e o IPPMG, com o objetivo de avaliar o conhecimento e a aceitação dessa população em relação à homeopatia. Através das respostas obtidas, foi possível traçar um perfil dos médicos entrevistados, caracterizando as duas populações entrevistadas quanto ao conhecimento da terapêutica homeopática. Dessa forma, o estudo apresenta uma primeira avaliação acerca da homeopatia, em dois hospitais, que apresentam destaque quanto à formação de novos médicos. De uma maneira geral, verificamos que os médicos entrevistados não conhecem e não utilizam a terapia homeopática, enfatizando a importância da inclusão do ensino de homeopatia nas graduações de medicina, em especial na Faculdade de Medicina da UFRJ, visto que uma parcela considerável dos médicos entrevistados cursou a faculdade de medicina na própria UFRJ.

Nosso trabalho nos leva a concluir, de maneira sucinta, um aparente descompasso ou um desconhecimento dos gestores em relação aos princípios que norteiam o Sistema Único de Saúde e as diretrizes e metas da Política Nacional de Práticas Integrativas e Complementares do SUS. A homeopatia, como uma dessas práticas, tem por premissa o princípio da integralidade do indivíduo (12), o tratamento do paciente como um todo, dentre outros aspectos contemplados na política do SUS. Além disso, verificamos a necessidade da terapêutica ser levada, de forma mais contundente, para dentro das instituições formadoras de médicos e outros profissionais da saúde, pois, sem profissionais especializados, será impossível implementar o serviço de homeopatia no SUS.

Trabalhos como este deveriam ser estimulados nas várias universidades e respectivos hospitais públicos brasileiros com o objetivo de mapear a percepção dos profissionais 
da saúde (médicos, farmacêuticos, enfermeiros e outros) acerca da terapêutica homeopática. A partir dos levantamentos, dados úteis poderão ser fornecidos aos gestores responsáveis pela implantação da homeopatia na Atenção Básica à Saúde, como previsto pela Política Nacional de Práticas Integrativas e Complementares do Ministério da Saúde.

\section{Referências}

1. Fontes OL. Farmácia Homeopática - Teoria e Prática. $4^{\mathrm{a}}$ ed. São Paulo: Editora Manole; 2013.

2. BRASIL. Farmacopeia Homeopática Brasileira. $3^{\mathrm{a}}$ ed., Brasília 2011.

3. Sales S. Presença da Homeopatia nas Faculdadesde Medicina Brasileiras: Resultados de uma Investigação Exploratória. Revista Brasileira de Educação 2008; 32(3): 283-290.

4. INSTITUTO DE PUERICULTURA E PEDIATRIA MARTAGÃO GESTEIRA. [acesso em ago de 2014]. Disponível em: http://ippmg.org.br/

5. HOSPITAL UNIVERSITÁRIO CLEMENTINO FRAGA FILHO. [acesso em ago de 2014]. Disponível em: http://www.hucff.ufrj.br/

6. Ribeiro M. História da Homeopatia no Brasil; 2010. [acesso em ago de 2014]. Disponível em: https://mariocabral.files.wordpress.com/2009/07/resumo-sobre-a-historia-da-homeopatia-no-brasilabril-2010.pdf

7. BRASIL. Ministério da Saúde. Gabinete do Ministro. Portaria $\mathrm{n}^{0}$ 971, de 3 de maio de 2006. Aprova a Política Nacional de Práticas Integrativas e Complementares no Sistema Único de Saúde. Brasília, 2006. Diário Oficial da União, Brasília, DF, DOU de 04 de mai. 2006, Seção 1, p. 64.

8. BRASIL. Ministério da Saúde. Gabinete do Ministro. Portaria n ${ }^{0}$ 849, de 25 de março de 2017. Inclui a Arteterapia, Ayurveda, Biodança, Dança Circular, Meditação, Musicoterapia, Naturopatia, Osteopatia, Quiropraxia, Reflexoterapia, Reiki, Shantala, Terapia Comunitária Integrativa e Yoga à Política. Diário Oficial da União, Brasília, DF, DOU de 28 de mar. 2017 nº 60, Seção 1, p. 68.

9. Manzato AJ, Santos AB. A Elaboração de Questionários na Pesquisa Quantitativa 2012. Disponível em:

http://www.inf.ufsc.br/ verav/Ensino_2012_1/ELABORACAO_QUESTIONARIOS_PESQUISA_Q UANTITATIVA.pdf

10. Scheffer MC, Cassenote AJFA. A feminização da medicina no Brasil. Revista Bioética 2013; 21(2):268-277.

11. Nascimento Mc, Romano V, Chazan Ac, Holandino C. Formação em Práticas Integrativas e Complementares em Saúde: Desafios para as Universidades Públicas. Trabalho, Educação e Saúde 2018; 16 (2): 751-772.

12. BRASIL. Ministério da Saúde. Secretaria Executiva. Sistema Único de Saúde (SUS): Princípios e Conquistas. Brasília, 2000. 